\title{
Playing Tag with HIF: The VHL Story
}

\author{
Sherri K. Leung and Michael Ohh* \\ Department of Laboratory Medicine and Pathobiology, Faculty of Medicine, \\ University of Toronto, Medical Sciences Building, Room 6306, \\ 1 King's College Circle, Toronto, Ontario, Canada M5S 1A8
}

Received 28 February 2002; accepted 13 May 2002

\begin{abstract}
Inactivation of the von Hippel-Lindau (VHL) tumour suppressor gene product pVHL is the cause of inherited VHL disease and is associated with sporadic kidney cancer. pVHL is found in a multiprotein complex with elongins B/C, Cul2, and Rbx1 forming an E3 ubiquitin ligase complex called VEC. This modular enzyme targets the $\alpha$ subunits of hypoxia-inducible factor (HIF) for ubiquitinmediated destruction. Consequently, tumour cells lacking functional pVHL overproduce the products of HIF-target genes such as vascular endothelial growth factor (VEGF), which promotes angiogenesis. This likely accounts for the hypervascular nature of VHL-associated neoplasms. Although pVHL has been linked to the cell-cycle, differentiation, and the regulation of extracellular matrix assembly, microenvironment $\mathrm{pH}$, and tissue invasiveness, this review will focus on the recent insights into the molecular mechanisms governing the E3 ubiquitin ligase function of VEC.
\end{abstract}

\section{INTRODUCTION}

Studies of tumour suppressor genes responsible for rare inherited cancer syndromes have revealed fundamental processes in cell biology as well as the molecular origins and pathogenesis of familial and sporadic forms of cancer. Germline mutations in the von Hippel-Lindau (VHL) tumour suppressor gene cause VHL disease, which is characterized by the development of tumours in multiple organs including the central nervous system (CNS), retina, adrenal gland, and kidney $[1,2]$. Although the incidence of VHL disease is infrequent with an approximate incidence of 1 in 40,000 individuals, bi-allelic inactivation of the VHL gene has been documented in a majority of sporadic cerebellar haemangioblastomas and renal clear cell carcinomas (RCC) [1]. $\mathrm{RCC}$ is the most common form of kidney cancer and is the main cause of death in VHL patients [3].

\section{CLONING OF THE VHL GENE}

Chromosome $3 p$ deletions are common in a variety of solid tumours including renal carcinomas $[4,5]$. Furthermore, several studies identified kindreds with early-onset, bilateral, and multifocal RCCs having germline translocations of $3 p$ to chromosomes 6,8 , or $11[6,7,8]$. In an effort to isolate the kidney cancer-susceptibility gene on chromosome $3 p$, scientists studied the hereditary kidney cancer associated with VHL disease, supposing that the VHL gene may be associated with the nonhereditary form of kidney cancer. In 1988, Seizinger and colleagues used genetic linkage analysis to map the VHL gene to a 6 to 8 centimorgan $(\mathrm{cM})$ region of chromosome 3 p25 to 3 p26 [9]. The putative VHL locus was then narrowed to a $4 \mathrm{cM}$ interval by multipoint linkage analysis, and pulse-field gel electrophoresis was subsequently used to further demarcate the minimal genomic region commonly deleted among unrelated VHL kindreds [10, 11]. Armed with this information, Latif and colleagues cloned the VHL gene in 1993 [12].

\section{VHL GENOTYPE-PHENOTYPE CORRELATIONS}

The detection of intragenic mutations that segregated with the disease in VHL kindreds confirmed the authenticity of the VHL gene. Furthermore, tumour-derived VHL gene mutations have been identified throughout the VHL open reading frame with the exception of the sequences encoding the first 54 amino acids $[1,2]$. Despite the mutational heterogeneity, striking correlations between certain germline mutations and specific clinical manifestations of the disease are emerging $[1,2]$. Such genotype-phenotype correlations have led to the classification of the VHL disease into subcategories depending on their propensity to develop phaeochromocytoma: Type 1 families have a low risk and Type 2 families have a high risk of developing phaeochromocytoma. Type 2 is further subdivided into 2a (low risk of renal carcinoma), $2 \mathrm{~b}$ (high risk of renal carcinoma), and $2 \mathrm{c}$ (develop exclusively phaeochromocytoma without the other stigmata of the disease). Germline mutations associated with Type 1 disease are usually deletions, microinsertions, and nonsense mutations, whereas those mutations associated with Type 2 disease are almost invariably missense mutations [13].

\section{VEC MULTIPROTEIN COMPLEX}

Neither the primary nucleotide nor the amino acid sequence of pVHL had any significant homology to proteins 


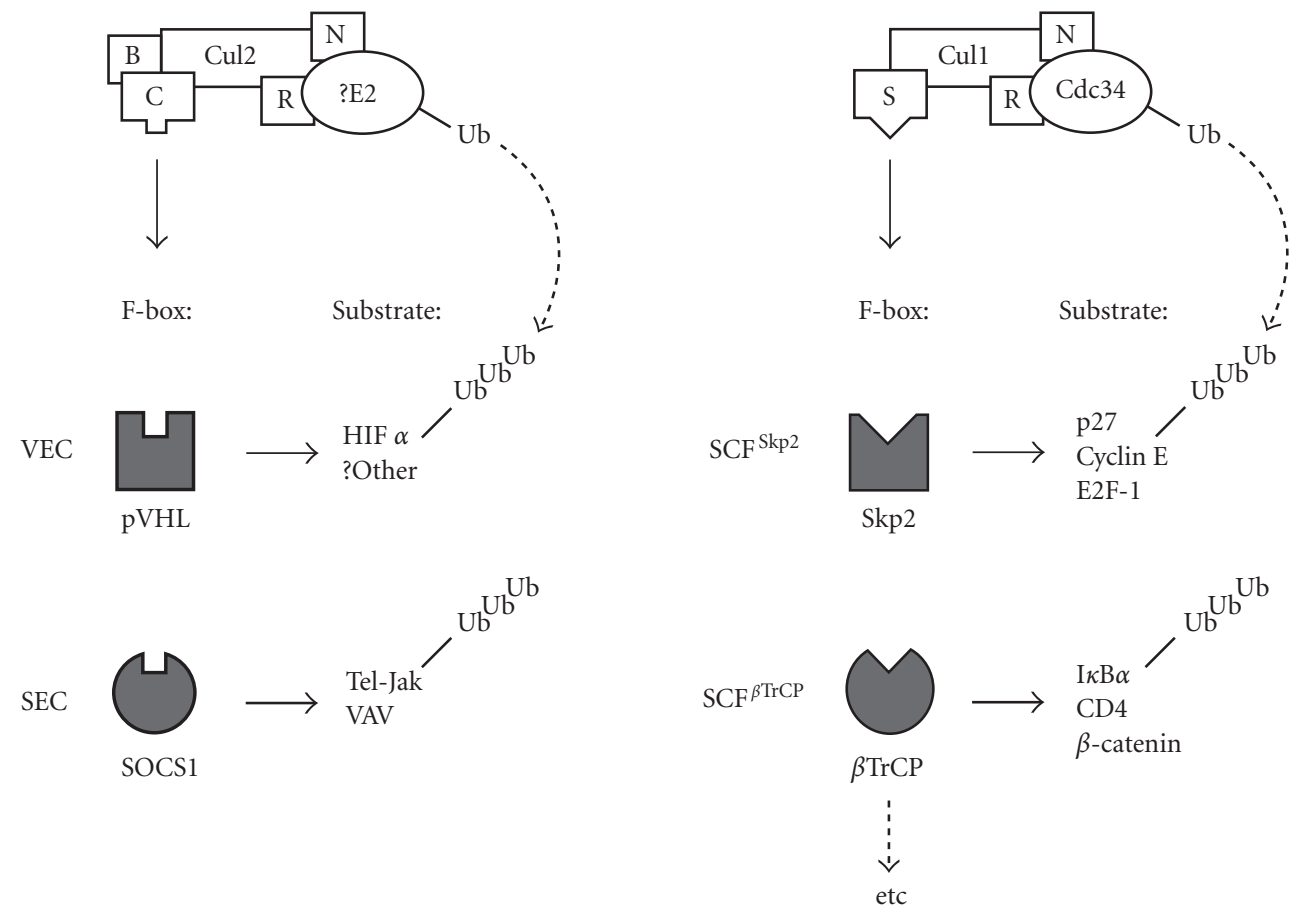

FIGURE 1. Comparison between the modular SCF and VEC complexes. SCF complex is composed of Skp1, Cdc53, Rbx1, and an F-box protein. Substrate specificity is conferred by the F-box protein. For example, $\mathrm{SCF}^{\text {Skp2 }}$ in concert with E2 ubiquitin-conjugating enzyme Cdc34 selectively targets the phosphorylated p27, as well as E2F-1 and Cyclin E, for ubiquitination. VEC complex is composed of Skp1-like elongin C, Cdc53 homologue Cul2, common Rbx1, ubiquitin-like elongin B and an F-box protein pVHL. The SCF-like VEC complex in concert with a cognate E2 targets prolyl hydroxylated HIF $\alpha$ subunits for ubiquitylation. NEDD8 conjugation to Cul1 or Cul2 enhances the E3 activity of both SCF and VEC complexes. Replacement of the F-box protein pVHL with SOCS1 forms SEC that specifically targets VAV or Tel-Jak. B represents elongin B; C represents elongin C; S represents Skp1; R represents Rbx1; N represents NEDD8.

with known functions. Thus, to discern the function(s) of pVHL, several groups independently sought to identify the cellular proteins that bound to pVHL, with the supposition that $\mathrm{pVHL}$-associated proteins might have identifiable functions. It is now evident that pVHL forms a multiprotein complex (VEC) with elongin B, elongin C, Cullin (Cul) 2, and Rbx1 (also called ROC1 or Hrt1) [14]. Remarkably, the VEC complex bears striking structural homology with a known class of E3 ubiquitin ligases called the SCF [14]. The E3 ubiquitin ligases target proteins for ubiquitylation, which marks them for proteolysis by the $26 \mathrm{~S}$ proteasome (see Figure 1). The SCF complex consists of Skp1 (S-phase kinase-associated protein 1 , suppressor of Cdk-inhibitor proteolysis and of kinetochore protein), Cdc53 or Cul1, Rbx1, and a substrate-conferring F-box protein (so called because of a collinear motif, first identified in cyclin F) (Figure 1).

Cul2 bears significant sequence and structural similarities to the yeast Cullin, Cdc53 [14]. Elongin C is homologous to Skp1 and performs an analogous Skp1-like role of bridging the F-box protein to the rest of the complex [14]. Rbx1 is a common component of VEC and SCF complexes [14]. The recently solved crystal structure of pVHL suggests that it might have a function analogous to that of an F-box protein [15]. Specifically, Pavletich and co-workers showed that pVHL has two domains: an $\alpha$ domain and a $\beta$ domain [15]. The $\alpha$ domain is essential for directly binding elongin $\mathrm{C}$, via the elongin $\mathrm{B} / \mathrm{C}$-binding motif, which loosely resembles the F-box. Elongin $\mathrm{C}$ is associated with elongin $\mathrm{B}$, Cul2, and Rbx1. Thus, pVHL binding to elongin $\mathrm{C}$ via the $\alpha$ domain promotes the nucleation of the VEC complex. The $\beta$ domain is predicted to function as a putative substratedocking site. Notably, disease-associated mutations found in VHL kindreds map to the surface residues of $\alpha$ and $\beta$ domains, suggesting the importance of both domains in the tumour suppressor function of pVHL. Thus, the structural homology between VEC and SCF complexes led to the notion that pVHL may have a role in targeting certain protein(s) for ubiquitin-mediated proteolysis (Figure 1).

\section{VHL DISEASE-ASSOCIATED TUMOURS AND VHL ANIMAL MODEL}

A vital clue to the potential downstream substrate or target of VEC came from the phenotypic observations of VHL disease-associated tumours. That is, tumours associated with VHL disease, such as retinal angiomas, cerebellar and spinal haemangioblastomas, and RCC, are known to be hypervascular [14]. This condition has been attributed primarily to the inability to suppress the expression of angiogenic peptides such as vascular endothelial growth factor (VEGF) that are normally induced by hypoxia $[1,14]$. In support of this observation, transformed cell lines lacking 
functional pVHL express elevated levels of VEGF even under normal oxygen tension $[1,2,14]$. Furthermore, Haase et al generated conditional VHL knockout mice using the CreloxP-mediated recombination, in which VHL can be inactivated in a spatially and temporally controlled manner [16]. $V H L$-/- mice are not viable, precluding the study of pVHL inactivation in adults [17]. Targeted disruption of VHL in the liver resulted in severe steatosis, numerous blood-filled vascular cavities, and foci of increased vascularization within the hepatic parenchyma [16]. Notably, hypoxia-inducible mRNAs were markedly increased [16]. Thus, the conditional mouse model for VHL disease underscores the significance of pVHL in the regulation of hypoxia-inducible genes.

\section{HIF, THE FIRST TARGET OF VEC}

A transcriptional complex called the hypoxia-inducible factor (HIF) is a global regulator of oxygen-dependent gene expression, including the aforementioned VEGF, which is upregulated during a cellular response to hypoxia $[18,19]$. HIF is a heterodimeric transcriptional activator, composed of $\alpha$ and $\beta$ subunits, that binds DNA in a sequence-specific manner. Whereas the $\alpha$ subunit is labile under normal oxygen tension, the $\beta$ subunit (also known as aryl hydrocarbon receptor nuclear translocator [ARNT]) is abundantly expressed independently of oxygen tension. The $\alpha$ subunit is subjected to ubiquitin-mediated degradation through a stretch of residues referred to as the oxygen-dependent degradation (ODD) domain. Hence, the regulation of HIF is through the control of the unstable $\alpha$ subunit $[18,19]$. Recently, Maxwell and colleagues observed that the $\alpha$ subunit is stabilized in cells devoid of wild-type pVHL [20]. More importantly, they found that HIF $\alpha$ subunits are physically associated with pVHL [20]. These observations suggested that the $\alpha$ subunits of HIF are the physiological targets of pVHL.

\section{TAGGING HIF WITH UBIQUITIN BY VEC}

HIF $\alpha$ subunits are observed to bind directly to the predicted $\beta$ domain of pVHL (Figure 1) [21, 22, 23]. Intriguingly, the ODD domain of HIF $\alpha$ subunit is both necessary and sufficient for binding to pVHL $[21,22,23]$. Furthermore, pVHL is specifically required for the ubiquitylation of HIF $\alpha$ subunits in vitro via the ODD domain $[21,22,23]$. Vascular tumour-derived mutations in the adapter $\alpha$ domain or the substrate-docking $\beta$ domain of pVHL abolished the ubiquitylation of $\mathrm{HIF} \alpha$ subunits, reiterating the concerted requirement of pVHL to form (1) a multiprotein ubiquitin ligase complex via elongin $\mathrm{B} / \mathrm{C}$-binding site within the $\alpha$ domain and (2) an efficient recognition of HIF $\alpha$ subunits via the $\beta$ domain [22]. Hypoxia mimetics attenuated the ubiquitin ligase activity of pVHL, reinforcing the notion that VEC targets HIF $\alpha$ under the selective presence of oxygen [22].

It is now known that in the presence of oxygen, iron, and oxoglutarate, $\mathrm{HIF} \alpha$ is hydroxylated at highly conserved proline residues, at positions 402 and $564[24,25,26]$.
Epstein and co-workers found that in Caenorhabditis elegans this process is accomplished by a novel EGL-9 dioxygenase [27]. There are three known mammalian EGL-9 homologues called PHD/HPH (prolyl hydroxylase domaincontaining protein, also referred to as HIF prolyl hydroxylase) 1,2 , and 3 , that perform an analogous function $[27,28]$. Importantly, hydroxylation of these proline residues is both sufficient and necessary for binding to pVHL $[24,25,26]$. Thus, under normal oxygen tension or normoxia, pVHL in an ubiquitin ligase complex selectively ubiquitylates the prolyl hydroxylated $\operatorname{HIF} \alpha[24,25,26]$. Importantly, the diseaseassociated pVHLs are unable to target the prolyl hydroxylated HIF $\alpha$ for ubiquitin-mediated proteolysis under normoxia [24]. Consequently, $\operatorname{HIF} \alpha$ subunits and the downstream targets of HIF transcription factor are overexpressed in cells lacking wild-type pVHL, irrespective of ambient oxygen tension. These observations establish pVHL as a vital component of a bona fide E3 ubiquitin ligase complex that marks prolyl hydroxylated HIF $\alpha$ subunits with a polyubiquitin chain for destructive targeting by the $26 \mathrm{~S}$ proteasome (see Figure 2).

It should be noted that prolyl hydroxylation as a posttranslational signal for recognition by an F-box protein represents the first exception to the current dogma of phosphorylation-dependent recognition of target proteins by the SCF complexes. Moreover, this raises the question of what other protein-protein interactions and biochemical processes are regulated by prolyl hydroxylation.

\section{MODULATION OF VEC FUNCTION BY NEDD8}

Despite our recent understanding of the oxygendependent pathway that controls HIF $\alpha$ recognition by VEC, the underlying mechanisms governing the actual activity of VEC remain unclear. Genetic experiments with Saccharomyces cerevisiae, Schizosaccharomyces pombe, and Arabidopsis thaliana showed that an ubiquitin-like protein Rub1 covalently modified Cdc53 and affected the function of SCF [29]. Likewise, the mammalian Rub-1 homologue NEDD8 is also conjugated to Cull in mammalian SCF and Cul2 in VEC $[30,31]$. The E2 NEDD8-conjugating enzyme, Ubc12, is required for the neddylation of Cullins including Cull and 2 $[32,33]$. Moreover, the neddylation of Cul1 or 2 increases the E3 ubiquitin ligase activities of SCF or VEC, respectively, in vitro $[34,35,36]$ and in vivo [34]. However, how Cul2 modification by NEDD8 enhances the E3 activity of VEC is unclear. One possibility is that NEDD8 conjugation to Cul2 increases the affinity of Cul2 to E2 ubiquitin-conjugation enzyme. In support of this notion, Kawakami and colleagues, recently demonstrated that NEDD8 conjugation to Cull enhances the affinity of SCF to the E2 ubiquitin-conjugation enzyme Ubc4 [37].

\section{THE “VHL-PATHWAY” AND FUTURE DIRECTIONS}

Recent insights into the molecular mechanisms governing the function of pVHL have provided significant 


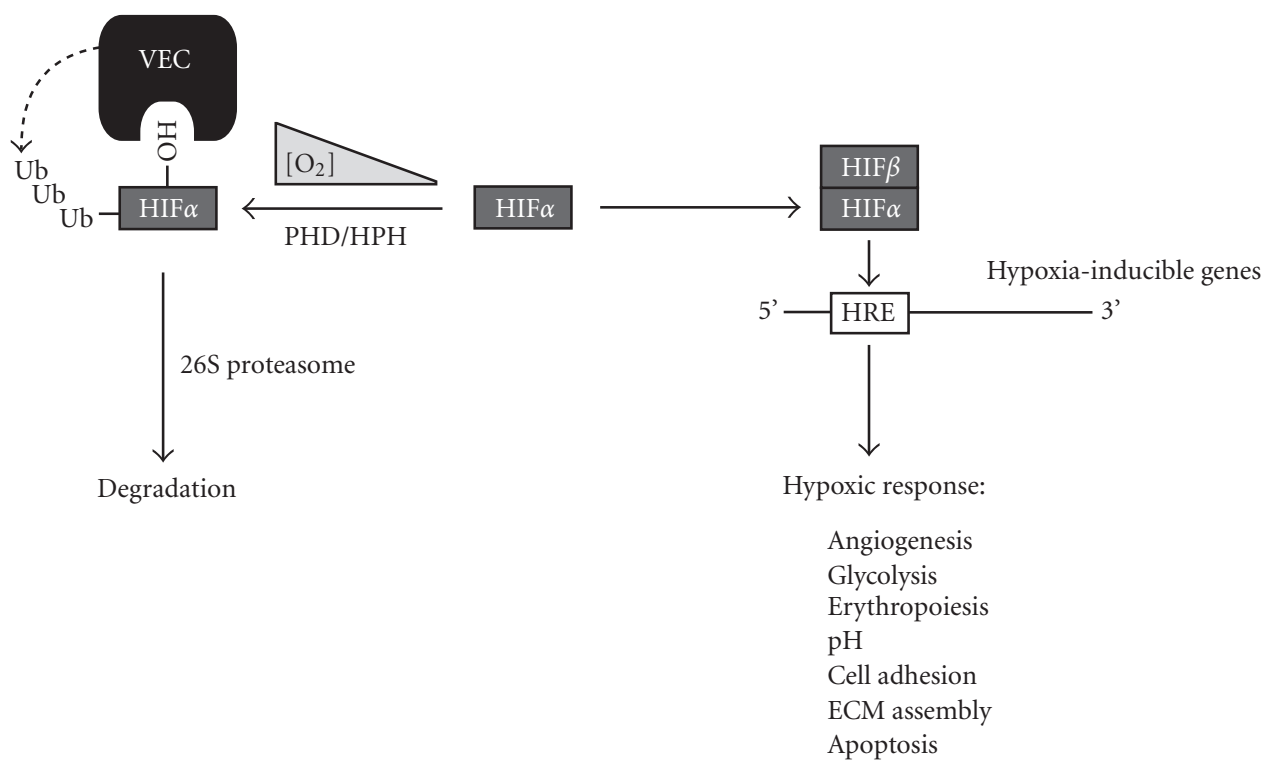

FIgURE 2. Oxygen-dependent gene regulation via the "VHL-pathway." In the presence of dioxygen, iron, and oxoglutarate, prolyl hydroxylases PHD/HPH 1,2, and 3 hydroxylate the $\alpha$ subunit of HIF on proline residues 402 and 564. The hydroxylated HIF $\alpha$ subunit is recognized by the VEC complex for ubiquitylation. In reduced oxygen tension, HIF $\alpha$ is no longer hydroxylated and thereby escapes ubiquitin-mediated proteolysis. HIF $\beta$ binds to the stable HIF $\alpha$ to form HIF, which promotes the transcriptional activation of numerous hypoxia-inducible genes (HIGs). Protein synthesis of HIGs results in various physiological responses to hypoxia. HRE represents hypoxia-responsive element; ECM represents extracellular matrix.

understanding of the "VHL-pathway." In the presence of cellular dioxygen, the newly identified dioxygenases, $\mathrm{PHD} / \mathrm{HPH}$ 1,2 , and 3 , selectively hydroxylate the evolutionarily conserved proline residues on HIF $\alpha$ subunits, which are then selectively recognized by VEC via pVHL $[27,28]$. VEC in concert with a cognate E2 ubiquitin-conjugating enzyme ubiquitylates the prolyl hydroxylated HIF $\alpha$ subunits for subsequent destruction by the $26 \mathrm{~S}$ proteasome. During hypoxia, HIF $\alpha$ is no longer modified by $\mathrm{PHD} / \mathrm{HPH}$ and consequently escapes ubiquitin-mediated proteolysis. The now stable $\operatorname{HIF} \alpha$ dimerizes with $\operatorname{HIF} \beta$ to form the transcription factor HIF, which binds to hypoxia responsive elements to trigger the transcriptional activation of numerous hypoxia-inducible genes, ultimately resulting in the synthesis of proteins that mediate the physiological responses to hypoxia (Figure 2). Thus, the "VHL-pathway" is intricately tied to the oxygen sensing mechanisms in multicellular organisms.

Further elucidation of the players that take part in the "VHL-pathway" will undoubtedly benefit the progress in the development of pharmacological agents to treat not only VHL patients but also those displaying tumours frequently associated with VHL disease, such as vascular tumours of the CNS and kidney. Moreover, the inability to maintain proper oxygen homeostasis is the one unifying the pathophysiology of stroke, cardiovascular diseases, chronic lung diseases, and cancer, which represent the most common causes of death in developed nations [38]. Thus, studies into the "VHLpathway" show great promise to aid in the design of synthetic drugs to block the progression of broad range of human diseases.

\section{ACKNOWLEDGMENTS}

We thank Drs Jeremy Mogridge, Douglas Templeton, and Meredith Irwin for helpful discussions. This work was supported by the National Cancer Institute of Canada with funds from the Terry Fox Run. MO is a Canada Research Chair in Molecular Oncology.

\section{REFERENCES}

[1] Maher ER, Kaelin WG Jr. von Hippel-Lindau disease. Medicine (Baltimore). 1997;76(6):381-391.

[2] Ohh M, Kaelin WG Jr. The von Hippel-Lindau tumour suppressor protein: new perspectives. Mol Med Today. 1999;5(6):257-263.

[3] Richards FM, Webster AR, McMahon R, Woodward ER, Rose S, Maher ER. Molecular genetic analysis of von Hippel-Lindau disease. J Intern Med. 1998;243(6):527533.

[4] Kovacs G, Erlandsson R, Boldog F, et al. Consistent chromosome $3 \mathrm{p}$ deletion and loss of heterozygosity in renal cell carcinoma. Proc Natl Acad Sci USA. 1988;85(5):1571-1575.

[5] Zbar B, Brauch H, Talmadge C, Linehan M. Loss of alleles of loci on the short arm of chromosome 3 in renal cell carcinoma. Nature. 1987;327(6124):721-724.

[6] Cohen AJ, Li FP, Berg S, et al. Hereditary renal-cell carcinoma associated with a chromosomal translocation. N Engl J Med. 1979;301(11):592-595.

[7] Kovacs G, Brusa P, De Riese W. Tissue-specific expression of a constitutional $3 ; 6$ translocation: development 
of multiple bilateral renal-cell carcinomas. Int J Cancer. 1989;43(3):422-427.

[8] Pathak S, Strong LC, Ferrell RE, Trindade A. Familial renal cell carcinoma with a $3 ; 11$ chromosome translocation limited to tumor cells. Science. 1982;217(4563):939-941.

[9] Seizinger BR, Rouleau GA, Ozelius LJ, et al. von Hippel-Lindau disease maps to the region of chromosome 3 associated with renal cell carcinoma. Nature. 1988;332(6161):268-269.

[10] Richards FM, Maher ER, Latif F, et al. Detailed genetic mapping of the von Hippel-Lindau disease tumour suppressor gene. J Med Genet. 1993;30(2):104107.

[11] Yao M, Latif F, Orcutt ML, et al. von Hippel-Lindau disease: identification of deletion mutations by pulsedfield gel electrophoresis. Hum Genet. 1993;92(6):605614.

[12] Latif F, Tory K, Gnarra J, et al. Identification of the von Hippel-Lindau disease tumor suppressor gene. Science. 1993;260(5112):1317-1320.

[13] Chen F, Kishida T, Yao M, et al. Germline mutations in the von Hippel-Lindau disease tumor suppressor gene: correlations with phenotype. Hum Mutat. 1995;5(1):66-75.

[14] Ivan M, Kaelin WG Jr. The von Hippel-Lindau tumor suppressor protein. Curr Opin Genet Dev. 2001;11(1):27-34.

[15] Stebbins CE, Kaelin WG Jr, Pavletich NP. Structure of the VHL-ElonginC-ElonginB complex: implications for VHL tumor suppressor function. Science. 1999;284(5413):455-461.

[16] Haase VH, Glickman JN, Socolovsky M, Jaenisch R. Vascular tumors in livers with targeted inactivation of the von Hippel-Lindau tumor suppressor. Proc Natl Acad Sci USA. 2001;98(4):1583-1588.

[17] Gnarra JR, Ward JM, Porter FD, et al. Defective placental vasculogenesis causes embryonic lethality in VHL-deficient mice. Proc Natl Acad Sci USA. 1997;94(17):9102-9107.

[18] Semenza GL. Perspectives on oxygen sensing. Cell. 1999;98(3):281-284.

[19] Semenza GL. HIF-1, O(2), and the 3 PHDs: how animal cells signal hypoxia to the nucleus. Cell. 2001;107(1):13.

[20] Maxwell PH, Wiesener MS, Chang GW, et al. The tumour suppressor protein VHL targets hypoxiainducible factors for oxygen-dependent proteolysis. $\mathrm{Na}$ ture. 1999;399(6733):271-275.

[21] Cockman ME, Masson N, Mole DR, et al. Hypoxia inducible factor-alpha binding and ubiquitylation by the von Hippel-Lindau tumor suppressor protein. J Biol Chem. 2000;275(33):25733-25741.

[22] Ohh M, Park CW, Ivan M, et al. Ubiquitination of hypoxia-inducible factor requires direct binding to the beta-domain of the von Hippel-Lindau protein. Nat Cell Biol. 2000;2(7):423-427.

[23] Tanimoto K, Makino Y, Pereira T, Poellinger L. Mech- anism of regulation of the hypoxia-inducible factor-1 alpha by the von Hippel-Lindau tumor suppressor protein. EMBO J. 2000;19(16):4298-4309.

[24] Ivan M, Kondo K, Yang $H$, et al. HIFalpha targeted for VHL-mediated destruction by proline hydroxylation: implications for $\mathrm{O} 2$ sensing. Science. 2001;292(5516):464-468.

[25] Jaakkola P, Mole DR, Tian YM, et al. Targeting of HIF-alpha to the von Hippel-Lindau ubiquitylation complex by O2-regulated prolyl hydroxylation. Science. 2001;292(5516):468-472.

[26] Masson N, Willam C, Maxwell PH, Pugh CW, Ratcliffe PJ. Independent function of two destruction domains in hypoxia-inducible factor-alpha chains activated by prolyl hydroxylation. EMBO J. 2001;20(18):5197-5206.

[27] Epstein AC, Gleadle JM, McNeill LA, et al. C. elegans EGL-9 and mammalian homologs define a family of dioxygenases that regulate HIF by prolyl hydroxylation. Cell. 2001;107(1):43-54.

[28] Bruick RK, McKnight SL. A conserved family of prolyl-4-hydroxylases that modify HIF. Science. 2001;294(5545):1337-1340.

[29] Hochstrasser M. Evolution and function of ubiquitinlike protein-conjugation systems. Nat Cell Biol. 2000;2(8):E153-E157.

[30] Ciechanover A, Orian A, Schwartz AL. Ubiquitinmediated proteolysis: biological regulation via destruction. Bioessays. 2000;22(5):442-451.

[31] Deshaies RJ. SCF and Cullin/Ring H2-based ubiquitin ligases. Annu Rev Cell Dev Biol. 1999;15:435-467.

[32] Gong L, Yeh ET. Identification of the activating and conjugating enzymes of the NEDD8 conjugation pathway. J Biol Chem. 1999;274(17):12036-12042.

[33] Liakopoulos D, Doenges G, Matuschewski K, Jentsch S. A novel protein modification pathway related to the ubiquitin system. EMBO J. 1998;17(8):2208-2214.

[34] Ohh M, Kim WY, Moslehi JJ, et al. An intact NEDD8 pathway is required for Cullin-dependent ubiquitylation in mammalian cells. EMBO Rep. 2002;3(2):177182.

[35] Podust VN, Brownell JE, Gladysheva TB, et al. A Nedd8 conjugation pathway is essential for proteolytic targeting of p27Kip1 by ubiquitination. Proc Natl Acad Sci USA. 2000;97(9):4579-4584.

[36] Read MA, Brownell JE, Gladysheva TB, et al. Nedd8 modification of cul-1 activates $\mathrm{SCF}^{\beta \mathrm{TrCP}}$-dependent ubiquitination of $\mathrm{I} \kappa \mathrm{B} \alpha$. Mol Cell Biol. 2000;20(7):23262333.

[37] Kawakami T, Chiba T, Suzuki T, et al. NEDD8 recruits E2-ubiquitin to SCF E3 ligase. EMBO J. 2001;20(15):4003-4012.

[38] Semenza GL. HIF-1 and human disease: one highly involved factor. Genes Dev. 2000;14(16):1983-1991.

\footnotetext{
* Corresponding author.

E-mail: michael.ohh@utoronto.ca

Fax: +1 4169785959
} 

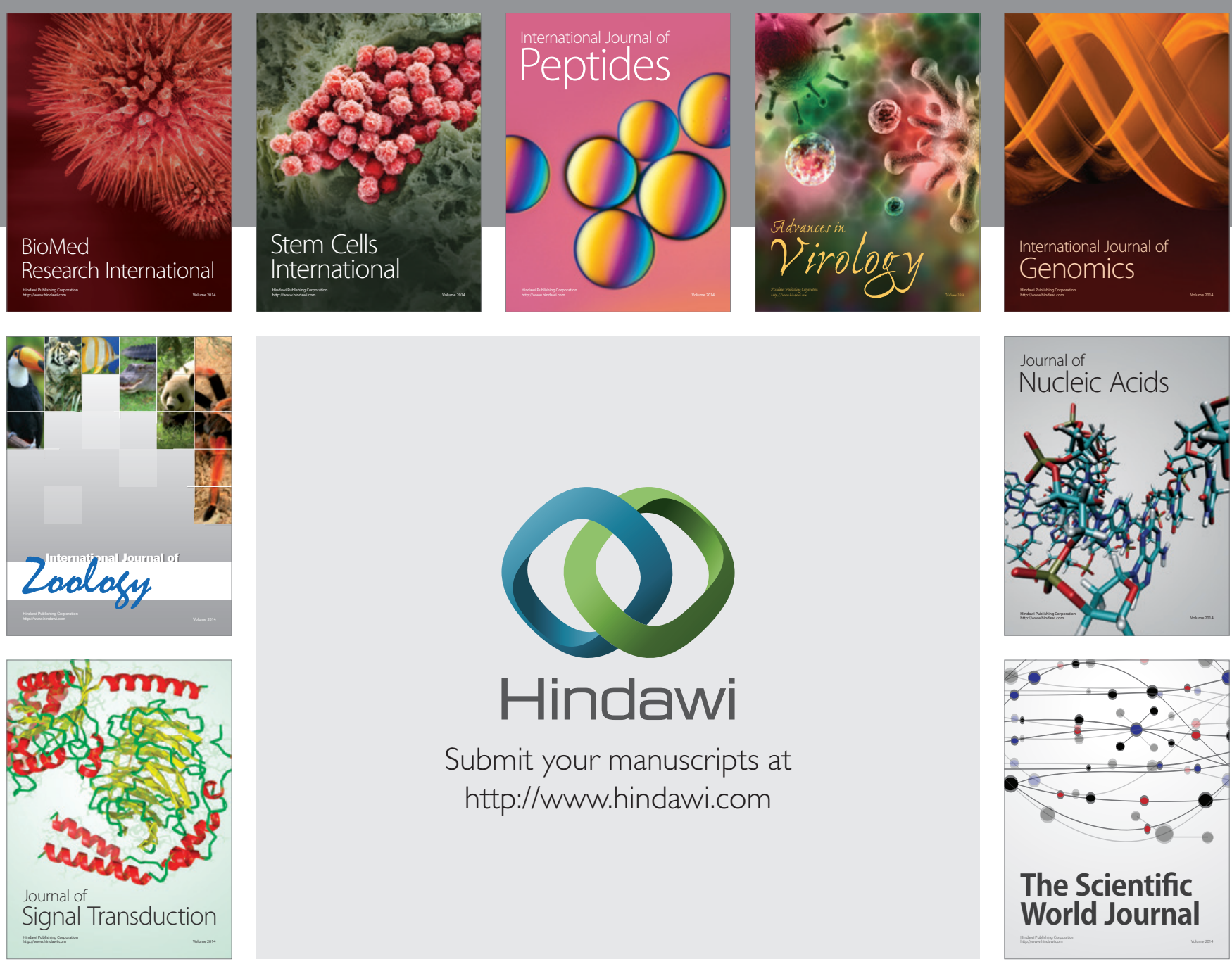

Submit your manuscripts at

http://www.hindawi.com
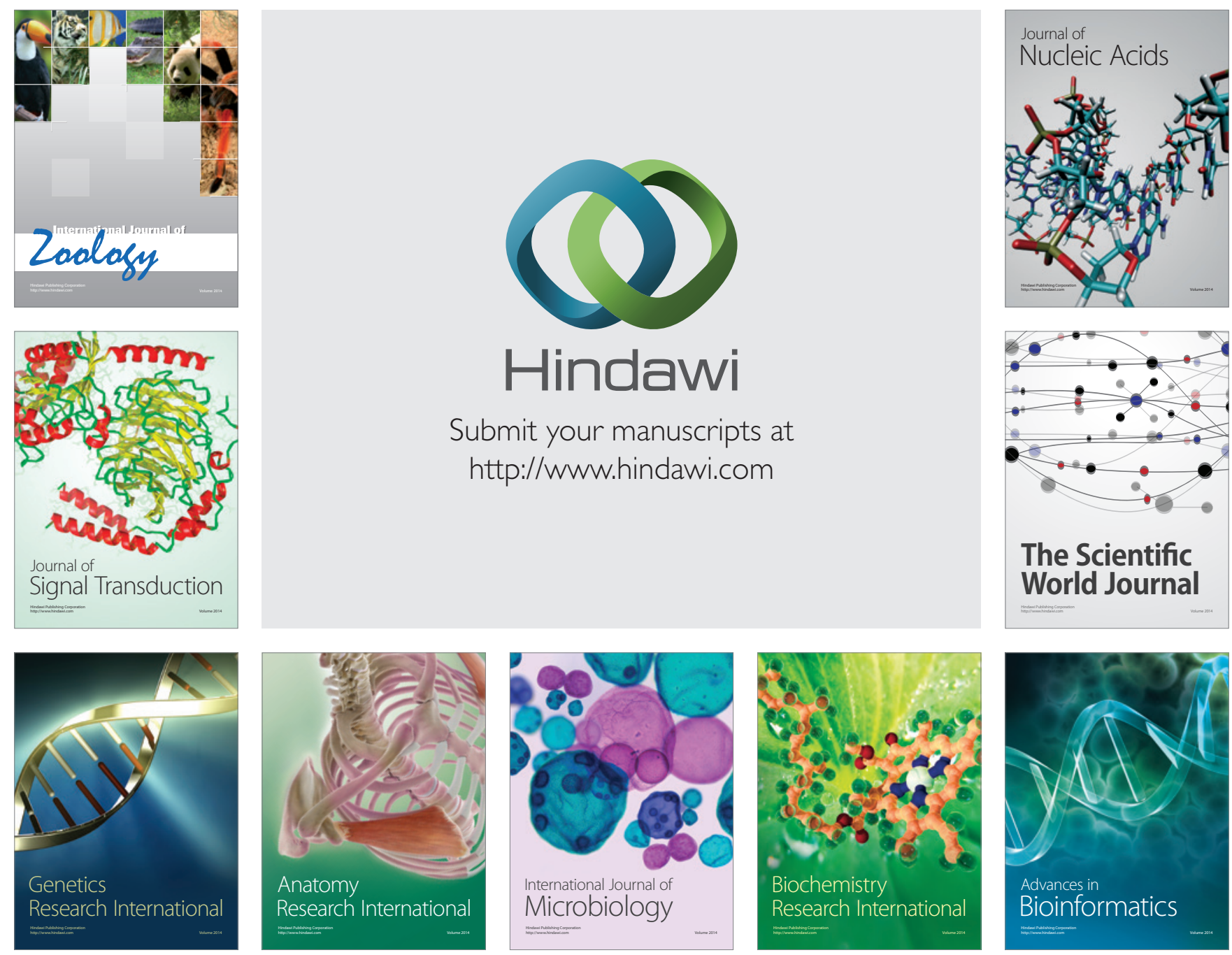

The Scientific World Journal
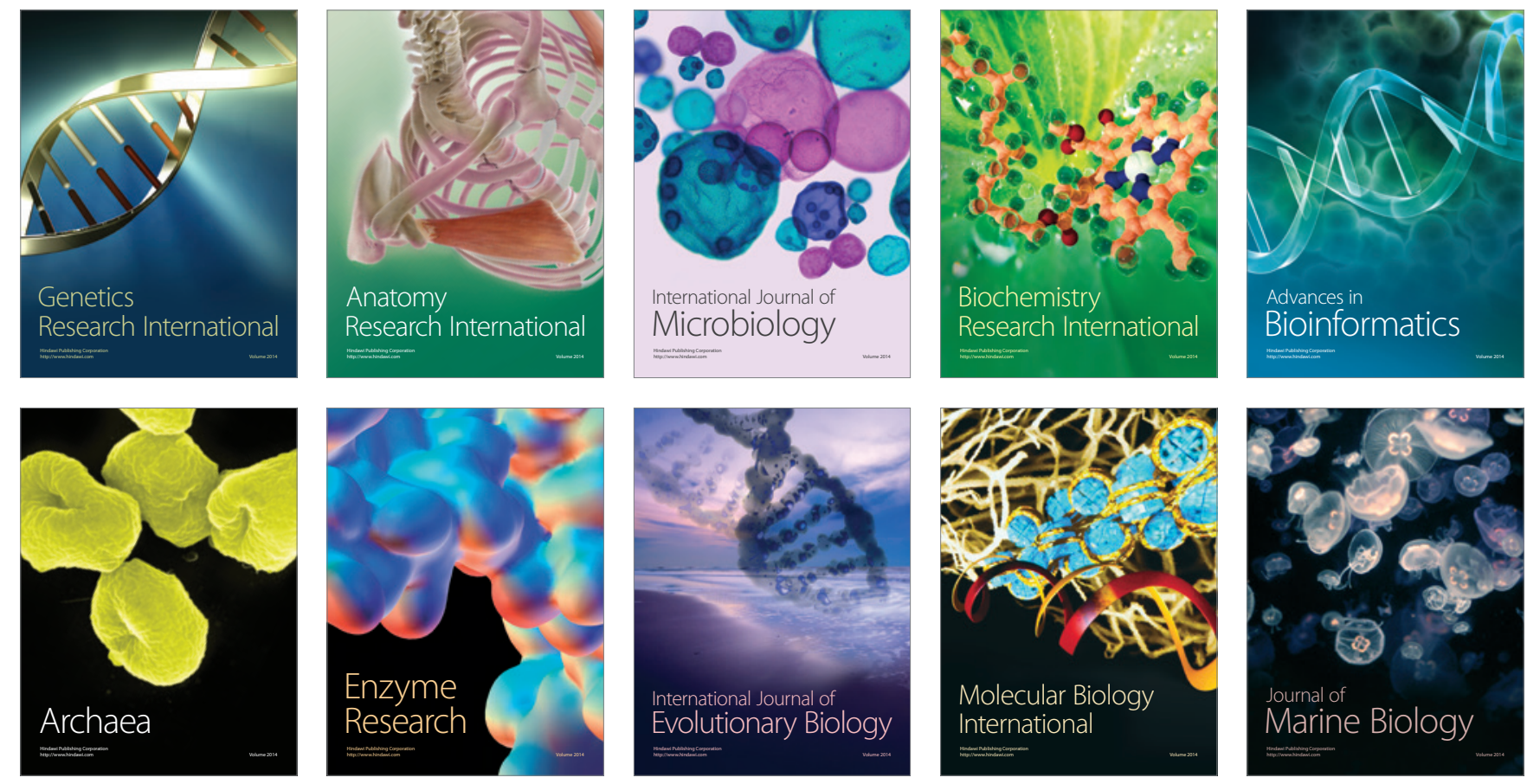